\title{
¿Qué hacemos ante un brote de escabiosis en un hospital?
}

\section{What we do in front of a scabies autbreak in a hospital settingn?}

\section{María Maestre Naranjo', Elena Martínez Fernández², María Fuentes Piñeiro², Rocío Gómez Grego³, Raquel García Arcones ${ }^{4}$, M. Luisa Rodríguez de la Pinta ${ }^{5}$}

1. Médico Adjunto. Servicio Prevención Hospital Universitario Puerta de Hierro Majadahonda.

2. Médico Residente. Servicio Prevención Hospital Universitario Puerta de Hierro Majadahonda.

3. Técnico Prevención. Servicio Prevención Hospital Universitario Puerta de Hierro Majadahonda-Sociedad de Prevención Fraternidad.

4. Enfermera. Servicio Prevención Hospital Universitario Puerta de Hierro Majadahonda.

5. Jefe Sección. Servicio Prevención Hospital Universitario Puerta de Hierro Majadahonda.

Recibido: 04-11-13

Aceptado: 24-03-14

\section{Correspondencia}

María Maestre Naranjo

Correo electrónico: mariaascension.maestre@salud.madrid.org

Resumen

La escabiosis o sarna es una enfermedad de la piel producida por Sarcoptes scabiei; puede ir aumentando el número de casos, hasta llegar a producir verdaderas epidemias ${ }^{1}$.

Se procede a describir las actuaciones del Servicio de Prevención de Riesgos Laborales de un hospital ante un brote de escabiosis ocurrido en una planta de hospitalización, con afectación de trabajadores sanitarios, tras el contacto con un paciente fuente (sospecha clínica y epidemiológica de escabiosis).

Med Segur Trab (Internet) 2014; 60 (234) 239-243

Palabras clave: escabiosis, enfermedad profesional, brote.

Abstract

The scabies is a skin disease produced by Sarcoptes scabiei; it has been an increase on the number of cases, reaching eventually an epidemic level.

We proceed to describe the measures taken by the laboral risk prevention service of a hospital against an outbreak of scabies happened in the hospitalization floor, with infection of the hospital workers after having contact with a source patient (clinic and epidemiologic scabies is suspected)

Med Segur Trab (Internet) 2014; 60 (234) 239-243

Keywords: scabies, Occupational disease, Outbreak 


\section{INTRODUCCIÓN}

La sarna o escabiosis es una enfermedad producida por la presencia en la piel del hombre del Sarcoptes scabiei, desarrollándose una reacción alérgica importante, cuyo principal síntoma es el picor ${ }^{1}$.

El reservorio es el hombre y la vía de transmisión es el contacto directo con la piel de una persona infestada. El parásito depende del huésped para vivir y fuera de la piel sólo sobrevive dos o tres días ${ }^{2}$.

El periodo de incubación (comprendido entre la infestación y la aparición de síntomas) sin exposición previa oscila entre 2 y 6 semanas, y en sensibilizados (infestación previa) puede ser desde horas hasta 4 días $^{3}$.

Existe una variante de la enfermedad denominada sarna noruega, que suele presentarse en personas de edad avanzada o con alteraciones inmunitarias, en la que los síntomas son más graves y existe un mayor riesgo de transmisión. El tratamiento de la sarna es sencillo, eficaz y poco tóxico ${ }^{1,2}$.

La escabiosis pertenece al Grupo III del cuadro de Enfermedades Profesionales: exposición a agentes biológicos y según RD 664/1997 sobre protección de los trabajadores contra los riesgos relacionados con la exposición a agentes biológicos durante el trabajo, el Sarcoptes scabiei hominis se clasifica en el grupo $2^{4,5}$.

Se entiende como brote un incremento significativo de casos en relación a los esperados $^{6}$. Destaca el subregistro de estas patologías en todo el Estado español si consideramos la incidencia de casos en Navarra. En el 2010, la declaración en Navarra fue de 677,5 por 100,000 trabajadores $^{12}$ frente a la declarada en España que fue de 116,21 por 100,000 trabajadores ${ }^{13}$.

\section{ANTECEDENTES}

Existe un paciente fuente con antecedentes de pápulas o vesículas. Ante la sospecha clínica y epidemiológica de escabiosis se inicia tratamiento con permetrina al 5\% presentando mala respuesta al tratamiento por lo que se realizan dos biopsias, con resultado negativo ( $\sin$ aislamiento en las muestras de Sarcoptes scabei). Tras mala evolución del cuadro y persistencia de la clínica se decide ingreso hospitalario para valoración y estudio de la sintomatología.

Se decide tratamiento con ivermectina ${ }^{8,9,10}$, iniciando una mejoría leve de la sintomatología. Se debe repetir dicho tratamiento en dos ocasiones más para remisión del cuadro. En ningún momento del proceso se procede al aislamiento de contacto del caso fuente, aunque algunos trabajadores, por propia iniciativa, utilizan medidas de protección cuando prestan asistencia a dicho paciente.

Durante la hospitalización, algunos trabajadores que prestan asistencia al paciente fuente presentan lesiones dérmicas, notificándolo a la supervisora de enfermería de la planta, quien lo comunica al Servicio de Prevención de Riesgos Laborales (SPRL), iniciándose en este momento el estudio correspondiente.

\section{Actuaciones del SPRL}

- El SPRL se coordina de forma inmediata con otros servicios del hospital para iniciar las medidas preventivas.

Se contacta con los Servicios de Dermatología, Medicina Preventiva, Farmacia, Dirección y se informa a las empresas de aquellos trabajadores que realizan su actividad en la planta afectada para que inicien las medidas preventivas adecuadas, según el artículo 24 de la Ley 31/1995 de Coordinación de actividades empresariales ${ }^{11}$. 
1. El Servicio de Dermatología realiza la valoración de las lesiones para el diagnóstico y establece las normas de tratamiento.

2. El Servicio de Medicina Preventiva establece las medidas de prevención y control de diseminación de la enfermedad entre los pacientes ingresados así como el estudio de contactos de los pacientes.

3. El Servicio de Farmacia Hospitalaria facilita el tratamiento indicado.

4. La Dirección es informada de lo ocurrido para que puedan tomar las medidas oportunas.

El SPRL inicia el estudio de contactos de las 29 personas (Tabla I) que desarrollaron sus tareas en esta planta de hospitalización durante el período en el que estuvo hospitalizado el paciente fuente, procediendo al registro de los contactos en una base de datos específica creada para tal fin. De ellos, siete trabajadores presentan lesiones en piel compatibles con escabiosis, siendo valorados por el servicio de dermatología confirmándose diagnóstico clínico y epidemiológico; se pauta tratamiento específico con permetrina al 5\% ${ }^{8,910}$, aplicándose el tratamiento desde el cuello hasta los pies y se deja actuar durante 12 horas. Transcurridos siete días se recomienda repetir el mismo tratamiento para asegurar la curación, en algún caso aislado se pauta tratamiento con antihistamínicos orales ${ }^{8,9,10}$.

Tabla I. Estudio de contactos

\begin{tabular}{lcccc}
\hline & CELADOR/TIGA & ENFERMERO/A & $\begin{array}{c}\text { AUXILIAR } \\
\text { ENFERMERÍA }\end{array}$ & TOTAL \\
\hline $\begin{array}{l}\text { ENFERMEDAD } \\
\text { PROFESIONAL }\end{array}$ & 1 & 3 & 3 & 7 \\
NO ENFERMEDAD & 1 & 9 & 12 & 22 \\
PROFESIONAL & 2 & 12 & 15 & 29 \\
TOTAL & & & & \\
\hline
\end{tabular}

Se recomienda a los convivientes de los trabajadores afectados que realicen simultáneamente la misma pauta de tratamiento; así como recomendaciones higiénicas en su entorno ${ }^{7,8}$.

Se facilita un informe a los trabajadores para sus Médicos de Atención Primaria (en nuestro caso la entidad colaboradora de la Seguridad Social en materia de Accidentes de trabajo y Enfermedades Profesionales es el Instituto Nacional de la Seguridad Social, prestando asistencia sanitaria a través de los médicos del Servicio Público de Salud) ${ }^{12}$ para que sea notificada la patología como Enfermedad Profesional ${ }^{4}$ y se facilite el parte de Incapacidad Temporal. El tiempo estimado de incapacidad temporal por este motivo es de un día, aunque varía en función de la clínica y evolución de cada caso.

De los siete trabajadores afectados, seis de ellos pertenecen al Servicio Madrileño de Salud y uno de ellos a una empresa subcontratada por la empresa concesionaria del hospital.

Los 22 trabajadores restantes no presentan lesiones, pero al estar en contacto con el paciente fuente o con sus compañeros afectados, realizan tratamiento profiláctico con permetrina $5 \%{ }^{7,8,9}$ para evitar el desarrollo y progresión de la enfermedad. En estos casos no se procede a notificación como Enfermedad profesional e Incapacidad temporal ${ }^{4}$. Es preciso destacar que hay dos trabajadoras embarazadas que no realizan tratamiento profiláctico por indicación del Servicio de Dermatología (aunque el tratamiento no presenta contraindicaciones ni para ellas ni para el feto).

Se informa a todos los trabajadores que ante cualquier incidencia deberán contactar urgentemente con el Servicio de Prevención. 
En paralelo el Servicio de Medicina Preventiva da indicaciones para la desinfección y limpieza de la habitación ocupada por el paciente fuente, así como del material, uniformes, utensilios y ropa de cama utilizadas ${ }^{13}$.

- El SPRL de acuerdo con lo dispuesto en el Art. 22 de la Ley 31/95 ${ }^{11}$ cita a los 30 trabajadores de la planta afectada para examen de vigilancia de la salud, tres o cuatro semanas tras el estudio de contactos. Se emiten 27 informes de Apto y dos Apto con limitaciones (de las dos trabajadoras embarazadas), relativas a su estado de gestación. Un trabajador de la planta afectada no acude a la cita del examen de salud por estar en Incapacidad Temporal en la fecha en que el caso fuente estuvo ingresado, así como en el momento de la cita, emitiéndose Informe de No Presentado.

- Otras actuaciones que se realizan por parte del SPRL son:

1. Revisión de la evaluación de riesgos laborales de la planta de hospitalización afectada, en cumplimiento de los requerimientos de la vigente Ley 31/1995 ${ }^{11}$ de Prevención de Riesgos Laborales y se obtienen los siguientes resultados.

1.1 Se observa que el protocolo de aislamiento no es bien conocido por las profesionales encargados de su puesta en funcionamiento, ya que el protocolo se activa en el momento en que se confirman los diagnósticos, cuando debería activarse ante la sospecha de la existencia de enfermedad. Se recomienda mejorar la difusión del actual protocolo de aislamiento, fundamentalmente por los médicos encargados de su puesta en marcha, tanto en las plantas de hospitalización como en el Servicio de Urgencias.

Escasa utilización de equipos de protección individual. Ante pacientes con sospecha de enfermedad infectocontagiosa de transmisión por contacto, se deberá utilizar de forma obligatoria para el acceso a la habitación o contacto con el enfermo, bata desechable que cubra los antebrazos y guantes de vinilo desechables ${ }^{13,14}$.

2. Se procede en coordinación con el Servicio de Medicina Preventiva a impartir un curso sobre "Medidas a adoptar ante sospecha-confirmación de enfermedad infecto-contagiosa". Se convocan a este curso todos los trabajadores de la planta y se imparte formación sobre medidas higiénicas y precauciones estándar. Además se da formación sobre actuación ante accidente de trabajo o enfermedad profesional, notificación de riesgos laborales, embarazo y lactancia, trabajadores especialmente sensibles y equipos de protección. Asisten 17 trabajadores, siendo registrados en la base de datos de formación del Servicio de Prevención.

\section{CONCLUSIÓN}

1. La coordinación de los servicios implicados es imprescindible para una actuación adecuada ante la aparición o contacto con un caso de escabiosis. El objetivo principal es garantizar la seguridad y salud de los trabajadores del Hospital, y evitar la diseminación de la enfermedad.

2. La elaboración de un protocolo de actuación conjunto entre los servicios de Dermatología, Medicina Preventiva y el Servicio de Prevención será de utilidad para el correcto manejo de nuevos casos de escabiosis.

3. Se debe tener a disposición de los trabajadores un procedimiento de actuación ante la sospecha de enfermedades infectocontagiosas y establecer procedimientos de comprobación del uso de los equipos de protección individual o elementos barrera.

4. Se deben impartir campañas específicas de información y/o sensibilización a los trabajadores sobre enfermedades transmisibles y la utilización adecuada de los equipos de protección.

5. Se debe declarar la escabiosis enfermedad profesional si existe nexo causal entre el caso fuente y los trabajadores afectados.

¿Qué hacemos ante un brote de escabiosis en un hospital? 


\section{BIBLIOGRAFÍA}

1. $\mathbf{M}^{\mathrm{a}}$. T. Campillos Páez, S. Causín Serrano, E. Duro Mota, S. Agudo Polo, M. O. Martínez Ramírez, J. M. Sánchez de la Nieta Martín Escabiosis: revisión y actualización Medifam v.12 n.7 Madrid jul. 2002.

2. Escabiosis: Principado de Asturias, Consejería de Salud y Servicios Sanitarios Sistema de Vigilancia Epidemiológica.

3. Gay Prieto J. Enfermedades parasitarias. En: Gay Prieto J, ed. Dermatología. 8 ${ }^{a}$ ed. Madrid: CientíficoMédica 1976.

4. Real Decreto 1299/2006, de 10 de noviembre, por el que se aprueba el cuadro de enfermedades profesionales en el sistema de la Seguridad Social y se establecen criterios para su notificación y registro. BOE-A-2006-22169.

5. Real Decreto 664/1997, de 12 de mayo, sobre la protección de los trabajadores contra los riesgos relacionados con la exposición a agentes biológicos durante el trabajo. BOE n 124 24-05-1997.

6. Real Decreto 2210/1995, 28 de Diciembre por la que se crea la Red Nacional de Vigilancia Epidemiológica. BOE núm 21, 24/01/1996.

7. Pediculosis-drugs therapy (drug consults). En: Gelman CR, et al, eds. Drugdex Information System. Englewood; Micromedex Inc, 1995.

8. Paasch U, Haustein UF. Treatment of endemic scabies with allethrin, permethrin and ivermectin. Evaluation of a treatment strategy. Hautarzt 2001; 52 (1): 31-7.

9. Madan V, Jaskiran K, Gupta U, Gupta DK. Oral invermectin in scabies patients: a comparison with $1 \%$ topical lindane lotion. J Dermatol 2001; 28 (9): 481-4.

10. Díaz-Maroto Muñoz, S.; Sarna y sarna noruega: diagnóstico, prevención y tratamientos actuales; Farm Hosp 1998; 22: 1-9.

11. Ley 31/1995, de 8 de noviembre, de Prevención de Riesgos Laborales, BOE n ${ }^{\circ} 269$, de 10 de noviembre.

12. Ley general de SS Real Decreto Legislativo 1/1994, de 20 de junio, por el que se aprueba el Texto Refundido de la Ley General de la Seguridad Social. BOE núm. 154 de 29 de Junio de 1994.

13. Alberto Larrosa Montañés, Manuel Cortés Blanco, Carlos Clerencia Casorrán Silvia Martínez Cuenca,Javier Urdániz Sancho,Julián Urbán Sender, Cristina Ariño Aldabo y Luis Güerri Mir. Brote de escabiosis en una residencia para personas mayores en la provincia de Huesca. Rev. Esp. Salud Pública vol. 78 n. 1 Madrid Jan. 2004.

14. Recomendaciones para la prevención, diagnóstico y tratamiento de la pediculosis y escabiosis. Ministerio de Salud, división, prevención y control de enfermedades Subsecretaría de Salud Pública. Departamento de Enfermedades Transmisibles. 